Universidad de Lima

Facultad de Psicología

Carrera de Psicología

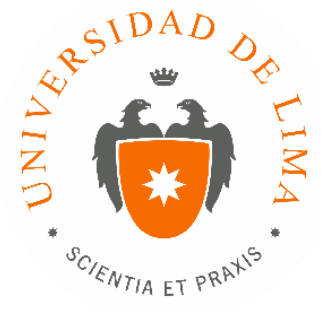

\title{
EXPERIENCIA DE TRABAJO EN EL DEPARTAMENTO DE PSICOLOGÍA DE UN PROYECTO SOCIAL EN SAN JUAN DE MIRAFLORES
}

Trabajo de suficiencia profesional para optar el título profesional de Licenciado en Psicología

Nicole Lindley Rocha

Código 20110658

Lima - Perú

Febrero de 2019 



\section{EXPERIENCIA DE TRABAJO EN EL DEPARTAMENTO DE PSICOLOGÍA DE UN PROYECTO SOCIAL EN SAN JUAN DE MIRAFLORES}




\section{TABLA DE CONTENIDO}

\section{INTRODUCCIÓN}

CAPÍTULO I: IDENTIFICACIÓN DEL PROBLEMA ….................................. 7

CAPÍTULO II: DESCRIPCIÓN DE LAS ACTIVIDADES REALIZADAS ...... 10

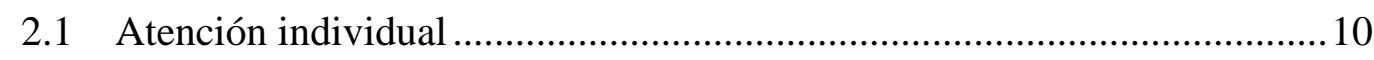

2.1.1 Evaluaciones..................................................................... 11

2.1.2 Consejería........................................................................... 12

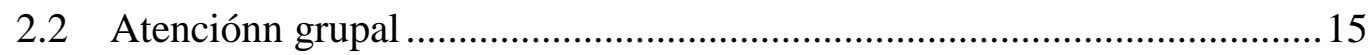

2.2.1 Taller de habilidades sociales................................................. 15

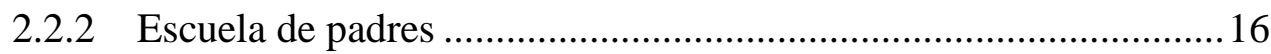

CAPÍTULO III: RESULTADOS DE LA INTERVENCIÓN ...............................17

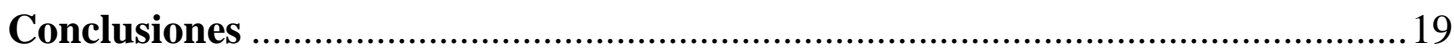

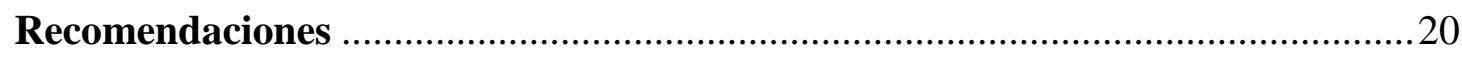

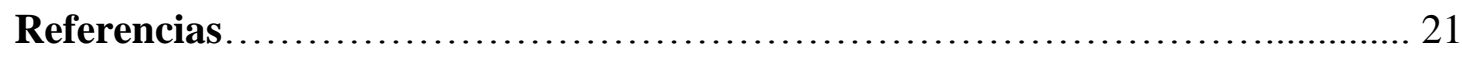

Apéndices.................................................................... 24 


\section{ÍNDICE DE APÉNDICES}

Apéndice 1: Taller de habilidades sociales................................................................ 25

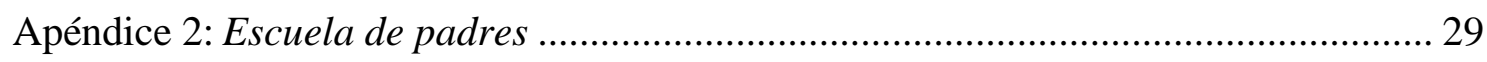

Apéndice 3: Esquema de informe psicológico .............................................................. 33 


\section{INTRODUCCIÓN}

Antiguamente se etiquetaba a las personas que asistían a psicoterapia como "locos" o “enfermos mentales" (Acuña, 2017). Sin embargo, gracias a estudios e investigaciones científicas, la psicología ha ido fortaleciendo su presencia en los últimos años, haciéndose más popular en los distintos continentes. A pesar de ello, aún se encuentran ciertos obstáculos en el camino, pues en ocasiones la información recibida por parte de la población es escasa o está sesgada, generando una difusión de falacias que afectan la reputación del rol del psicólogo y de las personas que asisten a terapia (Berrezueta y Vaca, 2018).

Esto, sumado a la baja oferta de servicios de salud mental dado que existe una falta de apoyo presupuestal por parte del gobierno (Ministerio de Salud, 2019) a pesar del gran porcentaje poblacional que padece o ha padecido trastornos mentales en algún momento de su vida, podría significar una limitación para el desarrollo profesional y bienestar personal de la comunidad (Piazza y Fiestas, 2014).

Es en este contexto que se crea el departamento de psicología como parte del proyecto social enfocado en apoyar a las personas de bajos recursos del distrito de San Juan de Miraflores. En el cual, las labores psicológicas de atención al público son de índole individual (evaluación y consejería) y/o grupal (talleres) según la necesidad de los consultantes. De este modo, gracias a los servicios brindados en el año 2017, se obtuvo como resultado una significativa cantidad de personas satisfechas con los mismos, incrementándose la demanda de solicitudes, así como también la psicoeducación a la comunidad sobre la psicología y sus principales funciones. 


\section{CAPÍTULO I: IDENTIFICACIÓN DEL PROBLEMA}

La presencia de los trastornos mentales desde hace décadas es una realidad en el Perú; sin embargo, no se le presta la atención suficiente, produciéndose un aumento de cifras significativo con el pasar de los años. En el 2014, se evidenció que el 29\% de la población en algún momento de su vida había padecido de una enfermedad mental, de los cuales el $90 \%$ no recibía atención psicológica (Piazza y Fiestas, 2014).

Cifras más recientes, brindadas por el Ministerio de Salud en el 2018, sugieren que 2 de cada 10 personas padecen de un trastorno mental al año, mientras que el 30\% de la población ha presentado uno en algún momento de su vida; siendo los más recurrentes los de ansiedad, control de impulsos, consumo de sustancias y del estado de ánimo. Sin embargo, a pesar de ello, el $80 \%$ no ha recibido ninguna atención por parte de los servicios de atención de salud pública. De esta forma, se puede observar que aún queda mucho trabajo por hacer, pues si bien ha disminuido la demanda atendida, las cifras continúan siendo alarmantes (Berríos, 2018).

De esta manera, los datos evidencian que existe una alta demanda y necesidad de atención psicológica por parte de la población para sobrellevar sus distintas dificultades y trastornos mentales (Berrezueta y Vaca, 2018). Que, al no ser abordados, se convierten en un obstáculo para lograr los objetivos de vida trazados, impide el desarrollo de estrategias de afrontamiento funcionales, disminuye la efectividad en las labores y el compromiso con la comunidad, por lo que pueden surgir conductas delictivas, frustración y más pobreza (Rondón, 2006).

En el Perú tanto la información sobre la importancia y el impacto de los trastornos de salud mental como las políticas con mira al bienestar de estos, son escasas; lo cual ha generado que con el pasar de los años haya ido en aumento la presencia de las enfermedades mentales (Alarcón, 2015; Hernández-Vásquez y Alhuay-Quispe, 2017). Las personas no tienen 
conocimiento sobre las sintomatologías y sus características, siendo un tercio de la población los que no identifican qué les sucede. Asimismo, se suele juzgar y estigmatizar a las personas que padecen de algún trastorno, otorgándole un significado erróneo, dando como resultado que se evite recurrir a un especialista de salud mental (Instituto Nacional de Salud Mental "Honorio Delgado - Hideyo Noguchi” [INSM], 2013).

Por otro lado, Bosetto (2006) afirma que la mayoría de las personas no conocen las funciones que realiza el psicólogo y por ello, tienden a recurrir a otras alternativas de solución antes de acudir a terapia, ya sea individual o grupal, pues no lo contemplan dentro de sus opciones principales. Lo cual se retroalimenta con el hecho de que las autoridades no consideren los servicios de salud mental como prioritarios en sus proyectos y deberes políticos (Pastor, 2008).

Además, el presupuesto que se asigna a los programas de prevención y atención de la salud mental en el Perú es significativamente inferior al de varios países de América Latina y por ende la oferta brindada por los profesionales de salud mental es escasa (Ministerio de Salud, 2019). Entonces, como consecuencia de esta falta de acceso a ciertos servicios y recursos, se presenta una vulnerabilidad social en la cual las personas se ven afectadas de forma individual y como sociedad, pues se generan también riesgos para la comunidad (Pizarro, 2001).

El proyecto social, es un programa de proyección social, médico y educativo, caracterizado por apoyar a las personas de bajos recursos del distrito de San Juan de Miraflores, en especial de la comunidad de Pamplona alta, Rinconada Alta y Nueva Rinconada. Brindando diversos servicios tales como educación básica alternativa (CEBA), centros de educación técnico productivo (CETPRO), médico, odontológico, asesoría legal y psicológica gratuita, comedores comunitarios, entre otros (PEBAL - La inmaculada, s.f.).

El centro se crea en 1978 por la fundación de un colegio privado, con la visión de ofrecer educación primaria a los pobladores de bajos recursos de la zona. Sin embargo, debido a las 
grandes necesidades insatisfechas, se fueron implementando nuevos programas y servicios para el beneficio de la comunidad (PEBAL - La inmaculada, s.f.). Es así como en 1995, la Universidad de Lima interviene, fundando el departamento psicológico; ofreciendo servicios de evaluación, orientación y consejería por parte de alumnos y docentes, el cual fue desarrollándose de acuerdo a las carencias percibidas (Universidad de Lima, s.f.).

Ahora bien, se evidencian dos problemas principales que presenta la población de Pamplona. El primero, es la falta de conciencia de lo que implica la salud mental, la importancia que se le debe otorgar, así como conocer el proceso terapéutico, pues una solicitud constante era la resolución inmediata de sus dificultades o las de su familia, que en significativas ocasiones al explicarles en qué consistía, evidenciaban su incomodidad y se generaban inasistencias. Sin embargo, y es aquí donde aparece el segundo problema, existía una alta demanda de atención psicológica, poca oferta y un bajo presupuesto con el que contaban las personas para poder cubrir con el gasto que implica llevar a cabo un proceso terapéutico, pues pertenecían a un nivel socioeconómico bajo. Razones por las cuales, se evidencia la gran necesidad de reforzar la oferta y facilitar la aproximación al sistema de salud mental pública, enfocándose en el desarrollo y ejecución de programas de prevención y atención de las distintas psicopatologías (Piazza y Fiestas, 2014). 


\section{CAPÍTULO II: DESCRIPCIÓN DE LAS ACTIVIDADES Y TAREAS REALIZADAS}

\subsection{Atención individual}

En el departamento psicológico del proyecto social se realizaba consejería y evaluaciones psicológicas a las personas que lo solicitaban. La atención era gratuita al ser un proyecto social y el rango de edades iba desde los 5 años hasta adultos mayores en el caso de la terapia, mientras que las evaluaciones no presentaban ningún criterio de edad.

El proceso consistía, en primer lugar, que las personas que requieran atención se presenten personalmente al departamento psicológico, con excepción de menores de edad. Luego, se les solicitaba un breve resumen de su motivo de consulta y la disponibilidad de horarios con la que contaban. Una vez establecido, en caso haya espacio en la agenda, se le asignaba una cita para la siguiente semana y se les explicaban las políticas del centro. Si la agenda estaba copada, se implementó una lista de espera en la cual se registraba el nombre de la persona solicitante, un breve motivo de consulta, el teléfono y, si el caso era delicado o prioritario se colocaba un símbolo para poder ser identificado con facilidad. Finalmente, una vez realizada la atención, se registraba en un cuadro de Excel los datos principales de la persona, incluyendo los posibles diagnósticos según el CIE-10 (Organización Mundial de la Salud [OMS], 1993), pues se estaba recolectando información con el fin de realizar un estudio epidemiológico en el distrito de San Juan de Miraflores orientado en identificar la presencia de trastornos mentales, así como también sus principales causas y problemáticas; cuyo aporte estaría dirigido hacia futuras investigaciones tanto de la zona como para la Universidad de Lima. 


\subsubsection{Evaluaciones}

Las evaluaciones se realizaban de dos maneras. La primera, utilizando el sistema DSM-IV (Morrison, Garduño, Enríquez y Larios, 2008) o CIE-10 (OMS, 1993), mediante la historia clínica y verificando el cumplimiento de la cantidad de criterios requeridos para el diagnóstico del trastorno o identificando los rasgos que pueda presentar la paciente dentro de alguno. Esta evaluación se efectuaba con la finalidad de identificar los posibles diagnósticos para establecer y planificar una mejor intervención considerando todas las características, así como también para derivar al psiquiatra en caso se considere necesario.

El segundo tipo de evaluaciones dependían de la solicitud del paciente, quien generalmente era el padre. Estas oscilaban entre evaluaciones cognitivas, perfiles psicológicos para el ingreso de los niños al colegio o nido y orientación vocacional, mientras que la cantidad de sesiones dependía de la edad del niño, su capacidad de atención y el nivel de agotamiento. Finalmente, una vez terminada la evaluación, el informe se entregaba en el transcurso de la siguiente semana, supervisado y firmado por el licenciado en psicología a cargo.

En todos los casos, se realizaba una entrevista inicial, con la finalidad de elaborar una anamnesis y recolectar información adicional a la arrojada por las pruebas y el motivo de consulta con mayor amplitud.

Las pruebas utilizadas para la realización del perfil psicológico eran principalmente tres: Escala de Maduración Social de Vineland, que examina la independencia y desenvolvimiento del niño en casa y fuera de ella (Balla, Cicchetti, D. y Sparrow, 1985), la Prueba de vocabulario de Peabody, 
encargada de medir la adquisición del vocabulario y la aptitud verbal (Dunn, Padilla y Lugo, 1986); y, por último, el Test de articulación del lenguaje Melgar adaptado, el cual identifica los descarríos de la articulación en los niños (Álvarez y Dionisio, 2010).

Por otro lado, en cuanto a las evaluaciones cognitivas, se utilizó el WISC IV Escala de inteligencia de Wechsler para niños, que evalúa el nivel de inteligencia general y en el área de comprensión verbal, razonamiento perceptivo, memoria de trabajo y velocidad de procesamiento (Wechsler y Corral, 2010). Sin embargo, cabe resaltar, la presente no se encuentra validada para la población a la que fue dirigida.

Finalmente, para la orientación vocacional se utilizaba un conjunto de pruebas encargadas de examinar distintas áreas del evaluado. La primera es el Big Five, que explora las características de personalidad del paciente (Barbarelli, Caprara y Borgogni, 1995), luego se realizaba una entrevista sobre sus intereses y preferencias junto con la prueba IPP Intereses y Preferencias Profesionales, que como su nombre lo refiere, se enfoca en los intereses de la persona considerando la profesión como tal y las tareas que se ejecutan dentro de esta (De la Cruz y López, 2000), y finalmente, el Test de Aptitudes mentales primarias PMA, que examina el nivel cognitivo del sujeto (Ugarriza y Palma, 1997).

\subsubsection{Consejería}

Las solicitudes de asistencia psicológica por parte de los pobladores eran diversas, oscilando entre dificultades para el manejo de su conducta y emociones, preocupaciones $\mathrm{y}$ en ocasiones trastornos mentales $\mathrm{y}$ de 
personalidad. Se pudo observar casos de depresión, ansiedad, trastorno de ansiedad generalizada, fobia social, trastorno de estrés post traumático, abuso de sustancias, trastorno límite de la personalidad (TLP), trastorno de pánico, dificultades en el control de impulsos, violencia doméstica, etc.

Debido a que los supervisores tenían distintas formaciones e inclusive más de una por persona, la orientación que se tenía era integrativa, pues se enseñaban y utilizaban técnicas Cognitivo-Conductuales, Contextuales de Aceptación y Compromiso (ACT) y Activación Conductual, y de la Terapia Dialéctica Conductual - DBT. Entonces, el plan de trabajo para la intervención con cada paciente dependía de diversas variables, como el objetivo terapéutico de cada uno, la capacidad de comprensión, la constancia, el trastorno mental en caso lo tuviera, las propias características de personalidad, entre otros. Aplicándose mediante el uso del modelo hipotético deductivo que se basa en escoger una técnica, emplearla y ver mediante la experiencia si es útil para la persona o necesita ciertas modificaciones (Baile, 2014).

Sin embargo, a pesar de las distintas técnicas, existía un patrón común en el proceso de la mayoría casos, con excepción de intervención en crisis. En la primera sesión de consejería, se llenaba un formato de ficha de registro que incluía datos de filiación y el motivo de consulta realizado mediante un análisis funcional de la conducta (Ruiz, Días y Villalobos, 2012). Asimismo, a lo largo de las sesiones se trabajaba empleando el uso constante de la validación en sus distintos niveles con la finalidad de crear y reforzar la relación de confianza con el paciente; así como también la psicoeducación, 
pues era de suma importancia que comprendan qué les sucedía, a qué podría deber y cómo sería el proceso.

A continuación, se detallarán las herramientas más utilizadas a lo largo de la experiencia de internado según cada corriente:

Las terapias de Tercera Generación o Contextuales consideran que los problemas o trastornos por los que padece la persona son originados por la interacción con el contexto en el que se desenvuelve. Es por ello que las estrategias empleadas hacen hincapié en la aceptación en lugar de la lucha por el cambio y se enfocan en guiar a las personas hacia la consecución de sus valores de manera que viva orientado hacia estos en el momento presente, en lugar de estancarse en su problema (Hayes, 2014). Las herramientas más utilizadas para ello fueron las de ACT y Activación Conductual. Con relación a la primera, se trabajó con la Matrix, defusión cognitiva, clarificación de valores, mindfulness y el uso de metáforas (Márquez-González, 2016). Por otro lado, se empleó el tratamiento breve de activación conductual para la depresión, que se enfoca en la modificación de patrones conductuales con el objetivo de motivar a la persona, hacerlo parte del proceso de mejora y orientarlos a encontrar su sentido de vida (Maero y Quintero, 2016).

Con respecto a las intervenciones Cognitivo-Conductuales, que consideran a los pensamientos como el eje de que determina la conducta y los sentimientos (Beriso, Plans, Sánchez-Guerra y Sánchez, 2003). Se utilizaron como técnicas el $\mathrm{ABC}$, el reconocimiento de distorsiones cognitivas, la reestructuración cognitiva, la búsqueda de evidencia la flecha invertida, los autorregistros, técnicas para ubicar metas, toma de decisiones y entrenamiento en relajación muscular progresiva (Ruiz et al., 2012). 
Por último, se emplearon herramientas de la DBT para ayudar al paciente a ser lo más efectivo posible dentro de su patología, cambiando sus patrones de conducta, pensamientos y emociones. Las estrategias más utilizadas fueron las de supervivencia en crisis, herramientas para regular emociones, el uso de contratos terapéuticos, evaluación de riesgo actual y efectividad interpersonal (Linehan, 2015).

\subsection{Atención grupal}

La realización de actividades terapéuticas grupales se ejecutaban de acuerdo a las necesidades observadas o solicitantes de atención psicológica, sobre la base de ello, se elaboraba un análisis de las mayores demandas que podrían trabajarse de manera grupal. Obteniendo dos resultados principales: una escuela de padres que enseñe como establecer límites de manera más efectiva y otro de habilidades sociales.

Cada taller era elaborado por dos internos del departamento psicológico, se realizaban investigaciones y recolectaba información que una vez obtenida, se coordinaba un espacio en el centro el cual se adapte a una mayor cantidad de personas y posterior a esto se hacía la publicidad del taller mediante folletos y carteles.

\subsubsection{Talleres de habilidades sociales}

Se realizó un taller de Efectividad Interpersonal de la corriente DBT, en el cual se trabajaron 3 módulos durante 4 sesiones una vez por semana. Estos incluían temas relacionados a la efectividad orientada hacia la consecución de una meta, la efectividad en las relaciones y la efectividad en el respeto por uno mismo. El objetivo del taller fue brindar estrategias a la comunidad de 
Pamplona para el manejo de las relaciones interpersonales, para aprender a equilibrar sus prioridades frente a demandas exigidas por los otros y generar sensación de competencia y respeto personal (Linehan, 2015).

\subsubsection{Escuela de padres}

Las escuelas para padres consistían en un taller de 4 sesiones una vez por semana, que se replicó en cuatro ocasiones debido a la alta demanda de padres que solicitaban atención para sus hijos pequeños desde los 2 años, pues referían que se comportaban mal, no seguían las indicaciones y consideraban que tenían serios problemas de conducta.

Los talleres consistían en brindar información clara, concisa y práctica a los padres sobre pautas de crianza, adaptada a su condición educativa, pues muchos de ellos solo contaban con primaria completa. Se trataban temas como los estilos de crianza más comunes, reacciones ineficaces y la comunicación efectiva, reforzadores y castigos tanto positivos como negativos y las distintas alternativas que tienen para aprender a manejar las conductas desafiantes de sus hijos. Además, se les enseñaba la técnica de economía de fichas con el objetivo de estructurar y organizar las normas en casa (Ruiz et al., 2012). Las explicaciones iban acompañadas de trabajos en grupo, conversatorios sobre sus experiencias personales, trabajos prácticos para la casa y juego de roles. Una vez finalizadas las sesiones, si los padres tenían más dudas o las dificultades en casa continuaban, se los atendía de forma individual. 


\section{CAPÍTULO III: RESULTADOS DE LA INTERVENCIÓN}

Durante el periodo laboral, se ofrecieron una serie de servicios enfocados en obtener la satisfacción de esa gran demanda existente debido al escaso alcance de los servicios de salud mental en el Perú, lo que significaba una mayor oferta (Piazza y Fiestas, 2014; Ministerio de Salud, 2019). Se brindó atención individual y grupal, adaptándose a las características y necesidades de cada trastorno o dificultad referida. De esta manera, gracias a la consejería brindada, se observó una disminución de obstáculos hacia la consecución de sus objetivos y una mayor dirección hacia sus valores, lo cual a su vez genera una satisfacción personal que los mismos pacientes describían durante las sesiones. Asimismo, se realizaron aproximadamente 10 evaluaciones psicológicas por cada interno, contribuyendo con el proceso de ingreso escolar de los niños, así como también, orientando en la toma de decisiones en cuanto a la carrera profesional o técnica a elegir.

En cuanto a la realización de los talleres, se pudo acceder a más personas debido a que estos se realizaron basados en las necesidades observadas y requeridas por los pacientes. Siendo la escuela para padres la que tuvo un mayor impacto en la población, pues por semana se acercaban 3 personas aproximadamente a solicitar atención para su hijo que no seguía indicaciones. Gracias a los talleres y la enseñanza de herramientas prácticas, al final del año disminuyeron estas demandas significativamente. De esta manera, durante el transcurso del año académico del 2017, se realizaron 4 escuelas para padres con un mínimo de asistencia de 8 personas por sesión.

Por otro lado, debido a la variedad de estrategias brindadas con base en distintos enfoques, se pudo ayudar de manera más efectiva a los pacientes, en un periodo menor a un año y con resultados positivos, pues se adaptaba la intervención de acuerdo a las necesidades y características de la persona, pudiendo utilizar distintas herramientas en un mismo paciente 
dependiendo del caso. Al usar las herramientas de las terapias Contextuales, se buscaba la aceptación del problema, eliminar el control de los pensamientos y dirigir a la persona hacia sus valores (Maero y Quintero, 2016), las Cognitivo-Conductuales se empleaban centrándose en los procesos cognitivos y su relación con lo emocional considerándose los mediadores en la perpetuación de la patología (Beriso et al., 2003) y finalmente, la DBT, se utilizaba en pacientes que necesitaban sentirse validados, pues su comportamiento tenía sentido en el contexto en el que se encontraban y se buscaba lograr un equilibrio con la modificación de la conducta (Linehan, 2015).

Asimismo, otro resultado favorecedor de la intervención es que se logró, en la medida de lo posible, brindar una mayor educación acerca de las funciones del psicólogo y la importancia de darle un lugar prioritario a la atención de la salud mental.

Los efectos mencionados, se evidenciaron mediante el agradecimiento brindado de manera explícita por parte de los pacientes, que hasta en ocasiones hacían un gran esfuerzo en tener detalles materiales con la intención de retribuir el apoyo. Así como también por el significativo aumento de solicitudes de atención psicológica por referencia de algún vecino, familiar o compañero. Lo cual llevó a la implementación de una lista de espera. A diferencia de años anteriores, que por la falta de pacientes se había tenido que repartir volantes por la zona para informar sobre la disponibilidad del servicio de atención psicológica.

No obstante, se observaron ciertas limitaciones. En ocasiones se evidenciaba resistencia a la terapia por parte de algunos pacientes, quienes incumplían con la resolución del material para casa y/o contaban con un número significativo de inasistencias o tardanzas, lo cual a su vez perjudicaba la efectividad de la intervención psicológica. Asimismo, la atención al público se veía obstaculizada, pues a pesar de contar con cuatro internos y algunos voluntarios, únicamente se contaba con tres consultorios, los cuales no daban abasto, produciéndose un aumento en la lista de espera. 


\section{CONCLUSIONES}

- La intervención individual utilizando estrategias de distintos enfoques dependiendo de las características personales, tuvo un impacto positivo en los pobladores de Pamplona Alta que asistían al departamento psicológico. Este resultado se obtuvo de primera mano, expresado por los mismos consultantes y observado en sus narrativas durante las sesiones.

- Las evaluaciones realizadas brindaron la información necesaria que permitió el ingreso de los niños a colegios y nidos. Así como también, se pudo orientar y guiar en cuanto a la vocación y profesión a elegir de las personas solicitantes.

- En cuanto a los talleres realizados, se pudo tener un acceso importante a los pobladores, brindándose información necesaria para la educación de sus hijos y en el manejo interpersonal.

- Debido al alcance que tuvieron los talleres y la consejería psicológica se pudo brindar una psicoeducación adecuada y realista a los vecinos de Pamplona de cuál es la función del psicólogo y se generó conciencia acerca de la importancia de darle un lugar prioritario a la salud mental.

- Las principales limitaciones durante el periodo laboral fueron las inasistencias a las sesiones ya programadas y la infraestructura del departamento de psicología.

- Por último, gracias aumento de la oferta en los servicios de atención a la salud mental brindados en el proyecto social, se pudo reducir parte de la demanda insatisfecha existente. 


\section{RECOMENDACIONES}

A continuación, se detallan las recomendaciones con respecto al centro laboral:

- Continuar con la organización y estructura de trabajo realizada, siguiendo con las atenciones tanto individuales como grupales, debido a que el tipo de problemática e intervención es distinto en cada caso.

- Brindar mayor posibilidad de horarios en cuanto a la realización de talleres grupales, así como también, incrementar su replicación. De modo que se obtenga un mayor alcance en la comunidad, que en ocasiones referían no poder asistir por motivos laborales.

- Aumentar la batería de pruebas psicológicas que estén validadas y actualizadas, con el objetivo de tener información más objetiva que refuerce la obtenida por medio de la observación de conducta, así como también que se adapte al tipo de población en la que se encuentra el centro.

- Contar con un día determinado cada cierto intervalo de tiempo en el que se dedique a realizar trabajo de campo; recolectando información y realizando charlas informativas acerca del rol del psicólogo, los principales trastornos mentales y el tipo de ayuda que podrían recibir en caso solicitarla.

- Implementar un sistema que aumente el compromiso con la terapia, caracterizado por brindar un número telefónico de referencia, ya sea de algún familiar o vecino, que avale su participación en los servicios y a la vez, sirva de fuente motivacional, de manera que disminuya el incumplimiento con las citas ya programadas.

- Por último, continuar con el estudio epidemiológico con la finalidad de contar con información objetiva de las principales dificultades y trastornos mentales dentro de la población de San Juan de Miraflores, así como también evidenciar la continua labor que se ejerce en el departamento de psicología. 


\section{REFERENCIAS}

Acuña, E. A. (2017). La psicoterapia: un momento oportuno para los psicólogos clínicos. Psicología desde el Caribe, 34(3). Recuperado de http://eds.b.ebscohost.com/eds/pdfviewer/pdfviewer?vid=0\&sid=5b1b5228-a1214d30-9ef2-0be8cbcd1ee1\%40pdc-v-sessmgr02

Alarcón, R. D. (2015). Salud Mental y Salud Pública en el Perú: ya es tiempo de actuar. Revista de Neuro-Psiquiatría, 78(1), 1-2. Recuperado de http://www.scielo.org.pe/scielo.php?script=sci_arttext\&pid=S0034$85972015000100001 \& \operatorname{lng}=\mathrm{es} \& \operatorname{lng}=\mathrm{pt}$.

Álvarez, R. G. y Dionisio, D. A. (2010). Programa RD y articulación verbal en niños y niñas de la IEP El Niño de Belén. (Tesis para optar el título de licenciada en Pedagogía y humanidades). Universidad Nacional del Centro del Perú. Recuperado de http://repositorio.uncp.edu.pe/bitstream/handle/UNCP/2509/Alvarez\%20RomoDionisio\%20Varillas.pdf? sequence $=1 \&$ is Allowed $=\mathrm{y}$

Baile, J. I. (2014). Intervención y tratamiento psicológico. Madrid: CEF.

Balla, D., Cicchetti, D. y Sparrow S. (1985). Vineland Behavior Scales-VINELAND, Escala de maduración social. Minnesota: American Guidance Service.

Barbarelli, C., Caprara, G. V. y Borgogni, L. (1995). BFQ Cuestionario “Big Five”. Madrid: TEA.

Beriso, A., Plans, B., Sánchez-Guerra, M. y Sánchez, D.I. (2003). Cuadernos de terapia cognitivo conductual: una orientación pedagógica e integradora. Madrid: Eos.

Berrezueta, L.F. y Vaca, J.F. (2018). Representación Social del Psicólogo Clínico en la población adulta de Cuenca-Ecuador. (Tesis para optar el título psicología)

Universidad de Cuenca, Ecuador. Recuperado de http://dspace.ucuenca.edu.ec/handle/123456789/30121

Berríos, M. (14 de febrero del 2018). El 80\% de peruanos con trastornos mentales no recibe tratamiento. La República. Recuperado de https://larepublica.pe/sociedad/1197071-el80-de-peruanos-con-trastornos-mentales-no-recibe-tratamiento

Bosetto, C.M. (2006). Reflexiones sobre la representación social de la psicología en un barrio de baja renta. Revista Diversitas, 2(1), 138-148. Recuperado de https://www.redalyc.org/html/679/67920110/

De la Cruz, M. V. y López, C. (2000). IPP: intereses y preferencias profesionales: Madrid. Tea.

Dunn, L., Padilla, E., Lugo, D. y Dunn, L. (1986). Manual del examinador para el Test de Vocabulario en Imágenes Peabody - Adaptación hispanoamericana. Minnesota: AGS

Fiestas, F. y Piazza, M. (2014). Prevalencia de vida y edad de inicio de trastornos mentales en el Perú urbano: Resultados del estudio mundial de salud mental, 2005. Revista 
Peruana De Medicina Experimental y Salud Pública, 31(1), 39-47. doi: 10.17843/rpmesp.2014.311.6

Hernández-Vásquez, R. y Alhuay-Quispe, J. (2017). Necesidad de promoción, prevención e intervención en salud mental en el Perú. Revista de Salud Pública, 19(2), 276. Recuperado de https://www.proquest.com/LATAM-ES/

Instituto Nacional de Salud Mental "Honorio Delgado - Hideyo Noguchi". (2013). Estudio epidemiológico de salud mental en Lima Metropolitana y Callao - replicación 2012. Informe general. Anales de Salud Mental, 19(1). Recuperado de http://www.insm.gob.pe/investigacion/archivos/estudios/2012\%20ASM\%20EESM\%20-LM.pdf

Linehan, M. M. (2015). DBT skills training manual (2nd ed.). New York: The Guilford Press.

Maero, F. y Quintero, P.J. (2016). Tratamiento breve de activación conductual para la depresión: protocolo y guía clínica. Buenos Aires: Akadia.

Márquez-González, M. (2016). Tendencias actuales en intervención psicológica. Madrid: Síntesis.

Ministerio de Salud. (2009). Informe sobre los servicios de Salud Mental del Subsector Ministerio de Salud del Perú 2008: Instrumento de Evaluación para Sistemas de Salud Mental de la Organización Mundial de la Salud (IESM-OMS). Recuperado de https://www.who.int/mental_health/mhgap/evidence/mh_who_aims_peru_apr2010_e n.pdf?ua $=1$

Morrison, J. R., Garduño, M., Enríquez, G. y Larios, P. (2008). DSM-IV: Guía para el diagnóstico clínico. México: Manual Moderno.

Organización Mundial de la Salud. (1993). CIE 10 trastornos mentales y del comportamiento: criterios diagnósticos de investigación. Madrid: Organización Mundial de la Salud.

Pastor, J. (2008). El psicólogo en atención primaria: un debate necesario en el sistema nacional de salud. Papeles del Psicólogo, 29 (3), 281-290. Recuperado de https://www.redalyc.org/html/778/77829304/

PEBAL - La Inmaculada. (s.f.). Historia PEBAL la Inmaculada. Recuperado de https://www.pebalsj.org/hitoria.html

Pizarro, R. (2001). La vulnerabilidad social y sus desafíos: Una mirada desde América Latina. Serie Estudios Estadísticos y Prospectivos, 6. CEPAL: Santiago de Chile. https://repositorio.cepal.org/bitstream/handle/11362/4762/S0102116_es.pdf?sequence $=1 \&$ isAllowed $=\mathrm{y}$

Rondón, M. B. (2006). Salud mental: un problema de salud pública en el Perú. Revista Peruana de Medicina Experimental y Salud Publica, 23(4), 237-238. Recuperado de http://www.scielo.org.pe/scielo.php?script=sci_arttext\&pid=S1726$46342006000400001 \& \operatorname{lng}=\mathrm{es} \& \operatorname{tlng}=\mathrm{es}$ 
Ruiz, M. A., Díaz, M. I. y Villalobos, A. (2012). Manual de técnicas de intervención cognitivo conductuales (2a ed.). Bilbao: Desclée de Brouwer.

Ugarriza, N. y Palma, S. (1997). Estandarización del Test de Aptitudes Mentales Primarias (PMA) en escolares de Lima Metropolitana. Lima, Perú: Universidad Ricardo Palma.

Universidad de Lima. (s.f.). Psicología: Pebal. Recuperado de http://www.ulima.edu.pe/pregrado/psicologia/pebal

Wechsler, D. y Corral, S. (2010). WISC-IV: escala de inteligencia de Wechsler para niñosIV. Madrid: Pearson. 


\section{APÉNDICES}




\section{APÉNDICE 1: TALLER DE HABILIDADES SOCIALES}

\begin{tabular}{|c|c|c|c|c|}
\hline $\begin{array}{l}\text { TIEMPO } \\
\text { Sesión } 1\end{array}$ & ACTIVIDAD & OBJETIVO & METODOLOGÍA & MATERIALES \\
\hline $5^{\prime}$ & $\begin{array}{l}\text { Presentación de } \\
\text { expositores y } \\
\text { actividades que se } \\
\text { realizarán en el taller }\end{array}$ & $\begin{array}{l}\text { Presentar las } \\
\text { actividades del taller, a } \\
\text { grandes rasgos. }\end{array}$ & $\begin{array}{l}\text { Cada expositor mencionará su } \\
\text { nombre y uno de ellos } \\
\text { nombrará las actividades que } \\
\text { se harán durante todo el taller }\end{array}$ & \\
\hline $10^{\prime}$ & $\begin{array}{l}\text { Entrega de etiquetas } \\
\text { con nombre }\end{array}$ & $\begin{array}{l}\text { Llevar un registro de } \\
\text { asistencia e ir } \\
\text { conociendo sus } \\
\text { nombres }\end{array}$ & $\begin{array}{l}\text { Se tomará lista a los } \\
\text { participantes del taller en un } \\
\text { registro de asistencia y } \\
\text { posteriormente se le hará } \\
\text { entrega de una etiqueta con } \\
\text { su nombre. }\end{array}$ & $\begin{array}{ll}- & \text { Lapicero } \\
- & \text { Plumones } \\
- & \text { Etiquetas } \\
\text { Hoja de } \\
\text { asistencia }\end{array}$ \\
\hline $10^{\prime}$ & $\begin{array}{l}\text { Dinámica de } \\
\text { integración }\end{array}$ & $\begin{array}{l}\text { Integrar a los } \\
\text { participantes y } \\
\text { hacerlos sentir con } \\
\text { más confianza. }\end{array}$ & & \\
\hline $20^{\prime}$ & $\begin{array}{l}3 \text { estilos de } \\
\text { comunicación }\end{array}$ & $\begin{array}{l}\text { Conocer los diferentes } \\
\text { estilos de } \\
\text { comunicación de } \\
\text { manera teórica y } \\
\text { práctica. }\end{array}$ & $\begin{array}{l}\text { En primer lugar, se presentará } \\
\text { información teórica acerca de } \\
\text { los diferentes estilos de } \\
\text { comunicación. Luego, se } \\
\text { observarán videos que } \\
\text { ejemplifican los distintos } \\
\text { estilos y se reflexionará sobre } \\
\text { ellos. Por último, los } \\
\text { participantes deberán marcar } \\
\text { con un aspa, las conductas, } \\
\text { pensamientos, etc. que suelen } \\
\text { utilizar. }\end{array}$ & $\begin{array}{ll}- & \text { PPT } \\
\text { - } & \text { Videos } \\
\text { - } & \text { Proyector } \\
\text { - } & \text { Laptop } \\
\text { - } & \text { Hojas } \\
& \text { impresas } \\
\text { - } & \text { Lapiceros } \\
\end{array}$ \\
\hline $40^{\prime}$ & $\begin{array}{l}\text { Asertividad en una } \\
\text { discusión. }\end{array}$ & $\begin{array}{l}\text { Enseñar a los } \\
\text { participantes a aplicar } \\
\text { la asertividad en } \\
\text { discusiones }\end{array}$ & $\begin{array}{l}\text { Se presentarán las estrategias } \\
\text { de manera teórica y luego, a } \\
\text { través de distintas situaciones } \\
\text { planteadas, los participantes } \\
\text { deberán realizar un juego de } \\
\text { roles solucionándolas, } \\
\text { utilizando las estrategias } \\
\text { enseñadas. Luego, algunos } \\
\text { voluntarios actuarán esas } \\
\text { situaciones frente a todos. } \\
\text { Además, al finalizar cada } \\
\text { situación ejemplificada, se } \\
\text { solicitará que cada uno cuente } \\
\text { una situación similar en la que } \\
\text { haya podido utilizar lo } \\
\text { realizado en el taller. }\end{array}$ & $\begin{array}{ll}- & \text { PPT } \\
\text { - } & \text { Videos } \\
\text { - } & \text { Proyector } \\
\text { - } & \text { Laptop } \\
\text { - } & \text { Hojas } \\
& \text { impresas }\end{array}$ \\
\hline $15^{\prime}$ & Actividad de cierre & Cerrar la sesión & Conversatorio & \\
\hline
\end{tabular}




\begin{tabular}{|c|c|c|c|c|}
\hline $\begin{array}{l}\text { TIEMPO } \\
\text { Sesión } 2\end{array}$ & ACTIVIDAD & OBJETIVO & METODOLOGÍA & MATERIALES \\
\hline $10^{\prime}$ & $\begin{array}{l}\text { Entrega de etiquetas } \\
\text { y recordatorio de } \\
\text { sesión pasada }\end{array}$ & $\begin{array}{l}\text { Llevar un registro } \\
\text { de asistencia y } \\
\text { recordar sesión } \\
\text { pasada }\end{array}$ & $\begin{array}{l}\text { Se tomará lista a los } \\
\text { participantes del taller en un } \\
\text { registro de asistencia y } \\
\text { posteriormente se le hará } \\
\text { entrega de una etiqueta con su } \\
\text { nombre. }\end{array}$ & $\begin{array}{ll} & \text { Lapicero } \\
\text { - } & \text { Plumones } \\
\text { - } & \text { Etiquetas } \\
\text { - } & \text { Hoja de } \\
& \text { asistencia }\end{array}$ \\
\hline $20^{\prime}$ & $\begin{array}{l}\text { Definición de la } \\
\text { efectividad en la } \\
\text { consecución de } \\
\text { objetivos y las } \\
\text { habilidades }\end{array}$ & $\begin{array}{l}\text { Informar a los } \\
\text { participantes } \\
\text { acerca de la } \\
\text { efectividad } \\
\text { interpersonal }\end{array}$ & $\begin{array}{l}\text { Presentación de un PPT con } \\
\text { teoría acerca de efectividad } \\
\text { interpersonal, efectividad } \\
\text { interpersonal en la } \\
\text { consecución de objetivos y las } \\
\text { habilidades necesarias para } \\
\text { lograrlos. Por último, se } \\
\text { presentarán dos ejemplos en } \\
\text { los que se utilicen las } 7 \\
\text { habilidades. }\end{array}$ & $\begin{array}{ll}- & \text { PPT } \\
- & \text { Proyector } \\
- & \text { Laptop } \\
- & \text { Hojas } \\
& \text { impresas } \\
- & \text { Lapiceros }\end{array}$ \\
\hline $70^{\prime}$ & $\begin{array}{l}\text { Explicar las } \\
\text { habilidades para la } \\
\text { efectividad en la } \\
\text { consecución de } \\
\text { objetivos }\end{array}$ & 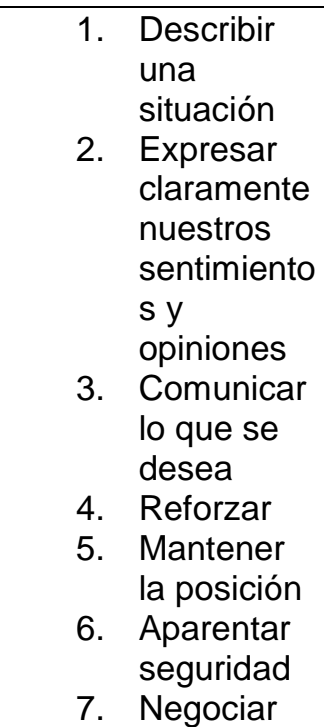 & $\begin{array}{l}\text { Mediante casos prácticos (role } \\
\text { - playing) se trabajarán estas } \\
7 \text { habilidades necesarias para } \\
\text { conseguir objetivos. }\end{array}$ & $\begin{array}{ll}- & \text { Casos } \\
\text { - } & \text { PPT } \\
\text { - } & \text { Proyector } \\
\text { - } & \text { Laptop } \\
\text { - } & \text { Hojas } \\
& \text { impresas } \\
\text { - } & \text { Lapiceros }\end{array}$ \\
\hline $10^{\prime}$ & $\begin{array}{l}\text { Conversatorio } \\
\text { respecto a las } \\
\text { actividades } \\
\text { anteriores y cierre }\end{array}$ & $\begin{array}{l}\text { Incentivar a que los } \\
\text { participantes se } \\
\text { expresen } \\
\text { verbalmente } \\
\text { respecto a sus } \\
\text { experiencias de la } \\
\text { actividad anterior. }\end{array}$ & $\begin{array}{l}\text { Sentarnos en una ronda, tanto } \\
\text { los participantes como los } \\
\text { expositores, y preguntar a } \\
\text { cada uno, cómo se sintieron y } \\
\text { por qué, respecto a la } \\
\text { actividad anterior. }\end{array}$ & \\
\hline
\end{tabular}




\begin{tabular}{|c|c|c|c|c|}
\hline $\begin{array}{l}\text { TIEMPO } \\
\text { Sesión } 3\end{array}$ & ACTIVIDAD & OBJETIVO & METODOLOGÍA & MATERIALES \\
\hline $10^{\prime}$ & $\begin{array}{l}\text { Entrega de } \\
\text { etiquetas y } \\
\text { recordatorio de } \\
\text { sesión pasada }\end{array}$ & $\begin{array}{l}\text { Llevar un registro } \\
\text { de asistencia y } \\
\text { recordar sesión } \\
\text { pasada }\end{array}$ & $\begin{array}{l}\text { Se tomará lista a los } \\
\text { participantes del taller en un } \\
\text { registro de asistencia y } \\
\text { posteriormente se le hará } \\
\text { entrega de una etiqueta con su } \\
\text { nombre. Asimismo, se hará un } \\
\text { breve repaso acerca de lo } \\
\text { visto en la sesión anterior. }\end{array}$ & $\begin{array}{ll}- & \text { PPT } \\
\text { - } & \text { Proyector } \\
\text { - } & \text { Laptop } \\
\text { - } & \text { Lapicero } \\
\text { - } & \text { Plumones } \\
\text { - } & \text { etiquetas } \\
\text { - } & \text { Hoja de } \\
& \text { asistencia }\end{array}$ \\
\hline $15^{\prime}$ & $\begin{array}{l}\text { Efectividad de las } \\
\text { relaciones }\end{array}$ & $\begin{array}{l}\text { Definir e informar } \\
\text { acerca de } \\
\text { efectividad para } \\
\text { mantener las } \\
\text { relaciones y sus } \\
\text { beneficios. }\end{array}$ & $\begin{array}{l}\text { Presentación de un PPT con } \\
\text { teoría acerca de efectividad } \\
\text { interpersonal para mantener } \\
\text { las relaciones, los beneficios y } \\
\text { la introducción de habilidades } \\
\text { para conseguirlo. }\end{array}$ & $\begin{array}{ll}- & \text { PPT } \\
\text { - } & \text { Proyector } \\
\text { - } & \text { Laptop }\end{array}$ \\
\hline $20^{\prime}$ & Ser agradable & $\begin{array}{l}\text { Enseñar a los } \\
\text { participantes a no } \\
\text { atacar, amenazar ni } \\
\text { juzgar a los demás }\end{array}$ & $\begin{array}{l}\text { Explicación teórica y role- } \\
\text { playing a partir de casos } \\
\text { particulares }\end{array}$ & $\begin{array}{ll}- & \text { PPT } \\
- & \text { Proyector } \\
\text { - } & \text { Laptop } \\
- & \text { Casos }\end{array}$ \\
\hline $20^{\prime}$ & $\begin{array}{l}\text { Interesarse por la } \\
\text { gente }\end{array}$ & $\begin{array}{l}\text { Enseñar a los } \\
\text { participantes a } \\
\text { mostrar interés por } \\
\text { los demás, } \\
\text { escuchando su } \\
\text { punto de vista y } \\
\text { razones. Además, } \\
\text { a no interrumpir y } \\
\text { respetar los deseos } \\
\text { del otro. }\end{array}$ & $\begin{array}{l}\text { Explicación teoría, video y } \\
\text { role-playing a partir de casos } \\
\text { particulares que se le } \\
\text { entregará a cada grupo de } 4\end{array}$ & $\begin{array}{ll}\text { - } & \text { PPT } \\
\text { - } & \text { Proyector } \\
\text { - } & \text { Laptop } \\
\text { - } & \text { Casos }\end{array}$ \\
\hline $20^{\prime}$ & Validar & $\begin{array}{l}\text { Enseñar a los } \\
\text { participantes a } \\
\text { reconocer los } \\
\text { sentimientos, } \\
\text { deseos, dificultades } \\
\text { y opiniones de las } \\
\text { otras personas sin } \\
\text { juzgarlas }\end{array}$ & $\begin{array}{l}\text { Entregar ejemplos con } \\
\text { opciones múltiples y circular la } \\
\text { opción en la que se dé la } \\
\text { validación. } \\
\text { Presentar casos particulares y } \\
\text { al terminar de leerlos plantear } \\
\text { una respuesta de validación } \\
\text { para luego comentarla frente a } \\
\text { los demás. }\end{array}$ & $\begin{array}{ll}\text { - } & \text { Ejemplos } \\
\text { - } & \text { Casos } \\
\text { - } & \text { Lapicero } \\
\text { - } & \text { PPT } \\
\text { - } & \text { Proyector } \\
\text { - } & \text { Laptop }\end{array}$ \\
\hline $15^{\prime}$ & $\begin{array}{l}\text { Ser simpático y } \\
\text { amable }\end{array}$ & $\begin{array}{l}\text { Enseñar a los } \\
\text { participantes a ser } \\
\text { alegre, sonreír y } \\
\text { tranquilizar a la otra } \\
\text { persona. }\end{array}$ & $\begin{array}{l}\text { Presentar videos que } \\
\text { ejemplifiquen dicha habilidad. }\end{array}$ & $\begin{array}{ll}\text { - } & \text { PPT } \\
\text { - } & \text { Proyector } \\
\text { - } & \text { Laptop }\end{array}$ \\
\hline $15^{\prime}$ & Cierre & $\begin{array}{l}\text { Incentivar a que los } \\
\text { participantes se } \\
\text { expresen } \\
\text { verbalmente } \\
\text { respecto a sus } \\
\text { experiencias de la } \\
\text { actividad anterior. }\end{array}$ & Conversatorio & \\
\hline
\end{tabular}




\begin{tabular}{|c|c|c|c|c|}
\hline $\begin{array}{l}\text { TIEMPO } \\
\text { Sesión } 4\end{array}$ & ACTIVIDAD & OBJETIVO & METODOLOGÍA & MATERIALES \\
\hline $10^{\prime}$ & $\begin{array}{l}\text { Entrega de } \\
\text { etiquetas y } \\
\text { recordatorio de } \\
\text { sesión pasada }\end{array}$ & $\begin{array}{l}\text { Llevar un registro } \\
\text { de asistencia y } \\
\text { recordar sesión } \\
\text { pasada }\end{array}$ & $\begin{array}{l}\text { Se tomará lista a los } \\
\text { participantes del taller en un } \\
\text { registro de asistencia y } \\
\text { posteriormente se le hará } \\
\text { entrega de una etiqueta con } \\
\text { su nombre. }\end{array}$ & $\begin{array}{ll}\text { - } & \text { Lapicero } \\
\text { - } & \text { Plumones } \\
\text { - } & \text { Etiquetas } \\
\text { - } & \text { Hoja de } \\
& \text { asistencia }\end{array}$ \\
\hline $15^{\prime}$ & $\begin{array}{l}\text { Efectividad } \\
\text { interpersonal en } \\
\text { cuanto al respeto } \\
\text { personal }\end{array}$ & $\begin{array}{l}\text { Definir e informar } \\
\text { acerca de } \\
\text { efectividad en } \\
\text { cuanto al respeto } \\
\text { personal }\end{array}$ & $\begin{array}{l}\text { Presentación de un PPT con } \\
\text { teoría acerca de efectividad } \\
\text { interpersonal en cuanto al } \\
\text { respeto personal, lo que } \\
\text { implica y la introducción de } \\
\text { habilidades para conseguirlo. }\end{array}$ & $\begin{array}{ll}- & \text { PPT } \\
- & \text { Proyector } \\
- & \text { Laptop }\end{array}$ \\
\hline $10^{\prime}$ & Ser justo & $\begin{array}{l}\text { Enseñar a los } \\
\text { participantes a ser } \\
\text { justos con uno } \\
\text { mismo y con los } \\
\text { demás }\end{array}$ & $\begin{array}{l}\text { Teoría y reflexión mediante } \\
\text { preguntas }\end{array}$ & $\begin{array}{ll}\text { - } & \text { Lista de } \\
& \text { preguntas } \\
\text { - } & \text { PPT } \\
\text { - } & \text { Proyector } \\
\text { - } & \text { Laptop }\end{array}$ \\
\hline $20^{\prime}$ & No disculparse & $\begin{array}{l}\text { Enseñar a los } \\
\text { participantes a } \\
\text { pedir disculpas } \\
\text { únicamente si es } \\
\text { necesario y está } \\
\text { justificado por } \\
\text { alguna } \\
\text { equivocación }\end{array}$ & $\begin{array}{l}\text { Diferentes casos y circular } \\
\text { aquellos que sean disculpas } \\
\text { justificadas. } \\
\text { Videos acerca de disculpas } \\
\text { injustificadas y justificadas } \\
\text { Conversatorio }\end{array}$ & $\begin{array}{ll}\text { - } & \text { Casos } \\
\text { - } & \text { Videos } \\
\text { - } & \text { PPT } \\
\text { - } & \text { Proyector } \\
\text { - } & \text { Laptop }\end{array}$ \\
\hline $20^{\prime}$ & $\begin{array}{l}\text { Mantener los } \\
\text { valores que } \\
\text { defendemos }\end{array}$ & $\begin{array}{l}\text { Enseñar a los } \\
\text { participantes a } \\
\text { defender su } \\
\text { postura ética y } \\
\text { moral }\end{array}$ & $\begin{array}{l}\text { Teoría de valores, cada } \\
\text { participante realizará una lista } \\
\text { acerca de sus valores } \\
\text { morales. } \\
\text { Videos antiéticos y como } \\
\text { responderían ante estos. }\end{array}$ & $\begin{array}{ll}\text { - } & \text { Videos } \\
\text { - } & \text { PPT } \\
\text { - } & \text { Proyector } \\
\text { - } & \text { Laptop } \\
\text { - } & \text { Hoja } \\
\text { - } & \text { Lapicero }\end{array}$ \\
\hline $20^{\prime}$ & Ser sincero & $\begin{array}{l}\text { Enseñar a los } \\
\text { participantes que } \\
\text { ser sinceros traerá } \\
\text { mejores resultados } \\
\text { a largo plazo }\end{array}$ & $\begin{array}{l}\text { Teoría acerca de las mentiras } \\
\text { y sus consecuencias. } \\
\text { Indagar acerca de } \\
\text { situaciones en las que hayan } \\
\text { mentido y les hayan mentido, } \\
\text { preguntar ¿cómo se } \\
\text { sintieron? } \\
\text { Metáforas (Fábulas) }\end{array}$ & $\begin{array}{ll}\text { - } & \text { PPT } \\
\text { - } & \text { Proyector } \\
\text { - } & \text { Laptop } \\
\text { - } & \text { Fábulas }\end{array}$ \\
\hline $20^{\prime}$ & $\begin{array}{l}\text { Conversatorio } \\
\text { respecto a las } \\
\text { actividades } \\
\text { anteriores y cierre }\end{array}$ & $\begin{array}{l}\text { Incentivar a que los } \\
\text { participantes se } \\
\text { expresen } \\
\text { verbalmente } \\
\text { respecto a sus } \\
\text { experiencias de la } \\
\text { actividad anterior. }\end{array}$ & $\begin{array}{l}\text { Sentarnos en una ronda, } \\
\text { tanto los participantes como } \\
\text { los expositores, y preguntar a } \\
\text { cada uno cómo se sintieron y } \\
\text { por qué, respecto a la } \\
\text { actividad anterior. }\end{array}$ & \\
\hline
\end{tabular}




\section{APÉNDICE 2: ESCUELA DE PADRES}

\begin{tabular}{|c|c|c|c|c|}
\hline $\begin{array}{l}\text { TIEMPO } \\
\text { Sesión } 1\end{array}$ & ACTIVIDAD & OBJETIVO & METODOLOGÍA & MATERIALES \\
\hline $5^{\prime}$ & $\begin{array}{l}\text { Tomar asistencia } \\
\text { Bienvenida al taller }\end{array}$ & $\begin{array}{l}\text { Llevar un registro de } \\
\text { asistencia e ir } \\
\text { conociendo sus } \\
\text { nombres }\end{array}$ & $\begin{array}{l}\text { Se tomará lista a los } \\
\text { participantes del taller en un } \\
\text { registro de asistencia y } \\
\text { posteriormente se le hará } \\
\text { entrega de etiqueta con su } \\
\text { nombre. }\end{array}$ & $\begin{array}{ll}\text { - } & \text { Lapicero } \\
\text { - } & \text { Plumones } \\
\text { - } & \text { Etiquetas } \\
\text { - } & \text { Hoja de } \\
& \text { asistencia }\end{array}$ \\
\hline $10^{\prime}$ & $\begin{array}{l}\text { Presentación de } \\
\text { expositores y } \\
\text { actividades que se } \\
\text { realizarán en el taller }\end{array}$ & $\begin{array}{l}\text { Presentar las } \\
\text { actividades del taller, a } \\
\text { grandes rasgos. }\end{array}$ & $\begin{array}{l}\text { Cada expositor mencionará } \\
\text { su nombre y uno de ellos } \\
\text { nombrará las actividades que } \\
\text { se harán durante todo el taller }\end{array}$ & \\
\hline $10^{\prime}$ & $\begin{array}{l}\text { Dinámica de } \\
\text { integración }\end{array}$ & $\begin{array}{l}\text { Integrar a los } \\
\text { participantes generando } \\
\text { confianza. }\end{array}$ & $\begin{array}{l}\text { Presentación individual, } \\
\text { edad, composición de familia } \\
\text { y exposición de las razones } \\
\text { por asistir al taller, }\end{array}$ & Pelota \\
\hline $30^{\prime}$ & $\begin{array}{l}\text { Dinámica del “Niño } \\
\text { interior” y compartir } \\
\text { según la experiencia } \\
\text { vivida y contención } \\
\text { psicológica. }\end{array}$ & $\begin{array}{l}\text { Recordar las } \\
\text { experiencias s que } \\
\text { ocurrieron durante la } \\
\text { crianza de los padres } \\
\text { presentes }\end{array}$ & $\begin{array}{l}\text { Actividad guiada de reflexión } \\
\text { individual sobre su niñez }\end{array}$ & \\
\hline $25^{\prime}$ & $\begin{array}{l}\text { Lluvia de ideas } \\
\text { grupales acerca de su } \\
\text { experiencia de crianza } \\
\text { en niñez }\end{array}$ & $\begin{array}{lr}\text { Elegir los } & \text { problemas/ } \\
\text { recuerdos } & \text { más } \\
\text { frecuentes } & \end{array}$ & $\begin{array}{l}\text { Cada representante de cada } \\
\text { grupo expondrá ante todas } \\
\text { las experiencias más } \\
\text { frecuentes }\end{array}$ & $\begin{array}{ll}- & \text { Paleógrafo } \\
- & \text { Plumones } \\
\text { Cinta catch } \\
\text { para pegar } \\
\text { paleógrafos } \\
\text { a pared }\end{array}$ \\
\hline $25^{\prime}$ & $\begin{array}{l}\text { Información acerca de } \\
\text { posibles alternativas al } \\
\text { castigo físico } \\
\text { Información sobre } \\
\text { acciones ineficaces en } \\
\text { crianza }\end{array}$ & $\begin{array}{lr}\text { Descubrir } & \text { conductas } \\
\text { alternativas al } & \text { golpe } 0 \\
\text { grito y aprender cuales } \\
\text { son las acciones } \\
\text { ineficaces } \\
\text { ejemplos. }\end{array}$ & Conversatorio & $\begin{array}{l}\text { Hojas } \\
\text { impresas } \\
\text { con } \\
\text { información }\end{array}$ \\
\hline $5^{\prime}$ & Actividad de cierre & Cerrar la sesión & $\begin{array}{l}\text { Ofrecer responder dudas } \\
\text { pendientes }\end{array}$ & \\
\hline
\end{tabular}




\begin{tabular}{|c|c|c|c|c|}
\hline $\begin{array}{l}\text { TIEMPO } \\
\text { Sesión } 2\end{array}$ & ACTIVIDAD & OBJETIVO & METODOLOGÍA & MATERIALES \\
\hline $5^{\prime}$ & $\begin{array}{l}\text { Entrega de etiquetas y } \\
\text { bienvenida al taller }\end{array}$ & $\begin{array}{l}\text { Llevar un registro de } \\
\text { asistencia e ir } \\
\text { conociendo sus } \\
\text { nombres }\end{array}$ & $\begin{array}{l}\text { Se tomará lista a los } \\
\text { participantes del taller en un } \\
\text { registro de asistencia y } \\
\text { posteriormente se le hará } \\
\text { entrega de una etiqueta con } \\
\text { su nombre. }\end{array}$ & $\begin{array}{ll}\text { - } & \text { Lapicero } \\
\text { - } & \text { Plumones } \\
\text { - } & \text { Etiquetas } \\
\text { - } & \text { Hoja de } \\
& \text { asistencia }\end{array}$ \\
\hline $10^{\prime}$ & $\begin{array}{l}\text { Dinámica para } \\
\text { recordatorio de sesión } \\
\text { pasada }\end{array}$ & $\begin{array}{l}\text { Refrescar material que } \\
\text { se aprendió en sesión } \\
\text { anterior }\end{array}$ & $\begin{array}{l}\text { Pedir a } 2 \text { voluntarios que de } \\
\text { manera alternada enumeren, } \\
\text { expliquen y ejemplifiquen las } \\
\text { acciones ineficaces en la } \\
\text { crianza mencionadas en la } \\
\text { sesión anterior }\end{array}$ & \\
\hline $20^{\prime}$ & $\begin{array}{l}\text { Exposición de distintos } \\
\text { estilos de crianza: } \\
\text { Autoritario - Permisivo } \\
\text { - Democrático } \\
\text { Y sus consecuencias }\end{array}$ & $\begin{array}{l}\text { Difundir los distintos } \\
\text { estilos parentales y sus } \\
\text { consecuencias en hijos }\end{array}$ & $\begin{array}{l}\text { Exposición mediante láminas } \\
\text { de PPT de los distintos estilos } \\
\text { parentales y las } \\
\text { consecuencias que tienen en } \\
\text { los hijos }\end{array}$ & $\begin{array}{ll} & \text { Laptop } \\
\text { - } & \text { USB - PPT } \\
\text { - } & \text { Proyector }\end{array}$ \\
\hline $30^{\prime}$ & $\begin{array}{l}\text { Juego de roles } \\
\text { ejemplificando los } \\
\text { estilos de crianza } \\
\text { enseñados }\end{array}$ & $\begin{array}{l}\text { Asegurarse de la } \\
\text { comprensión } \\
\text { interiorización de los } \\
\text { conceptos acerca de } \\
\text { estilos parentales de } \\
\text { crianza y consecuencias } \\
\text { de estos }\end{array}$ & $\begin{array}{l}\text { Los padres asistentes se } \\
\text { organizarán en } 3 \text { grupos e } \\
\text { idearán una dinámica } \\
\text { representativa de cada estilo, } \\
\text { ejemplificando acciones del } \\
\text { padre, madre y del hijo en } \\
\text { cada caso }\end{array}$ & \\
\hline $10^{\prime}$ & $\begin{array}{l}\text { Debatir cuál estilo de } \\
\text { crianza es el adecuado }\end{array}$ & $\begin{array}{l}\text { Identificar el estilo de } \\
\text { crianza adecuado y con } \\
\text { cuál fueron criados }\end{array}$ & $\begin{array}{l}\text { Se debatirá con los } \\
\text { participantes cuál es el estilo } \\
\text { de crianza más adecuado e } \\
\text { identificar cuál es con el que } \\
\text { ellos han crecido y si están de } \\
\text { acuerdo o no }\end{array}$ & $\begin{array}{l}\text { - Laptop } \\
\text { - PPT } \\
\text { - Proyector }\end{array}$ \\
\hline $15^{\prime}$ & $\begin{array}{l}\text { Exposición general } \\
\text { sobre reforzadores y } \\
\text { castigos }\end{array}$ & $\begin{array}{l}\text { Brindar información } \\
\text { acerca de cómo reforzar } \\
\text { y castigar }\end{array}$ & Exposición & $\begin{array}{l}\text { - Laptop } \\
\text { - PPT } \\
\text { - Proyector }\end{array}$ \\
\hline $15^{\prime}$ & $\begin{array}{l}\text { Exponer la importancia } \\
\text { del trabajo en equipo } \\
\text { entre la familia }\end{array}$ & $\begin{array}{l}\text { Concientizar sobre la } \\
\text { importancia del trabajo } \\
\text { en equipo en la crianza }\end{array}$ & $\begin{array}{l}\text { Explicar la importancia sobre } \\
\text { el trabajo en equipo familiar, } \\
\text { el estar de acuerdo con la } \\
\text { crianza de los hijos. }\end{array}$ & $\begin{array}{l}\text { - Laptop } \\
\text { - PPT } \\
\text { - Proyector }\end{array}$ \\
\hline $15^{\prime}$ & $\begin{array}{l}\text { Compartir lo elaborado } \\
\text { y cierre de la sesión }\end{array}$ & $\begin{array}{l}\text { Realizar resumen oral } \\
\text { acerca del tema tratado }\end{array}$ & $\begin{array}{l}\text { Se plantean algunas } \\
\text { preguntas a los asistentes } \\
\text { para asegurarse de la } \\
\text { comprensión del tema }\end{array}$ & \\
\hline
\end{tabular}




\begin{tabular}{|c|c|c|c|c|}
\hline $\begin{array}{l}\text { TIEMPO } \\
\text { Sesión } 3\end{array}$ & ACTIVIDAD & OBJETIVO & METODOLOGÍA & MATERIALES \\
\hline $5^{\prime}$ & $\begin{array}{l}\text { Entrega de etiquetas y } \\
\text { bienvenida al taller }\end{array}$ & $\begin{array}{l}\text { Llevar un registro de } \\
\text { asistencia e ir } \\
\text { conociendo sus } \\
\text { nombres }\end{array}$ & $\begin{array}{l}\text { Se tomará lista a los } \\
\text { participantes del taller en un } \\
\text { registro de asistencia y } \\
\text { posteriormente se le hará } \\
\text { entrega de una etiqueta con } \\
\text { su nombre. }\end{array}$ & $\begin{array}{ll}\text { - } & \text { Lapicero } \\
\text { - } & \text { Plumones } \\
\text { - } & \text { Etiquetas } \\
\text { - } & \text { Hoja de } \\
& \text { asistencia }\end{array}$ \\
\hline $10^{\prime}$ & $\begin{array}{l}\text { Dinámica para } \\
\text { recordatorio de sesión } \\
\text { pasada }\end{array}$ & $\begin{array}{l}\text { Refrescar material que } \\
\text { se aprendió en sesión } \\
\text { anterior }\end{array}$ & $\begin{array}{l}\text { Pedir a voluntarios } \\
\text { mencionen los estilos } \\
\text { crianza más comunes. }\end{array}$ & \\
\hline $10^{\prime}$ & $\begin{array}{l}\text { Exposición sobre qué } \\
\text { es reforzar }\end{array}$ & $\begin{array}{l}\text { Brindar información } \\
\text { acerca de qué es } \\
\text { reforzar. }\end{array}$ & Exposición & $\begin{array}{ll}- & \text { Laptop } \\
\text { - } & \text { PPT } \\
\text { - } & \text { Proyector } \\
\end{array}$ \\
\hline $20^{\prime}$ & $\begin{array}{l}\text { Exponer tipos de } \\
\text { refuerzo }\end{array}$ & $\begin{array}{l}\text { Comprender los tipos } \\
\text { de refuerzo }\end{array}$ & $\begin{array}{l}\text { Exponer los dos tipos de } \\
\text { refuerzo, positivo y negativo. }\end{array}$ & $\begin{array}{ll}\text { - } & \text { Laptop } \\
\text { - } & \text { PPT } \\
\text { - } & \text { Proyector }\end{array}$ \\
\hline $20^{\prime}$ & $\begin{array}{l}\text { identificar conductas } \\
\text { se deben reforzar y } \\
\text { cómo }\end{array}$ & $\begin{array}{l}\text { Comprender los tipos } \\
\text { de recompensa }\end{array}$ & $\begin{array}{l}\text { Exponer el tipo de conductas } \\
\text { más comunes que se deben } \\
\text { reforzar y los tipos de } \\
\text { recompensa para cada } \\
\text { conducta. }\end{array}$ & $\begin{array}{ll}\text { - } & \text { Laptop } \\
\text { - } & \text { PPT } \\
\text { - } & \text { Proyector }\end{array}$ \\
\hline $20^{\prime}$ & $\begin{array}{l}\text { Actividad individual: } \\
\text { aprendiendo a reforzar }\end{array}$ & $\begin{array}{l}\text { Asignar conductas que } \\
\text { les gustaría reforzar en } \\
\text { sus hijos }\end{array}$ & 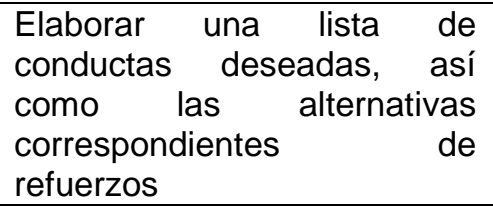 & $\begin{array}{ll}\text { - } & \text { Hojas } \\
\text { - } & \text { Lapiceros }\end{array}$ \\
\hline $20^{\prime}$ & $\begin{array}{l}\text { Trabajo en equipo: } \\
\text { resolución de casos }\end{array}$ & $\begin{array}{l}\text { Identificar que } \\
\text { reforzadores utilizarían } \\
\text { en distintas situaciones }\end{array}$ & $\begin{array}{l}\text { Se forman grupos y se les dan } \\
\text { dos casos en los cuales tienen } \\
\text { que identificar que reforzador } \\
\text { utilizar y a qué tipo } \\
\text { corresponden. }\end{array}$ & $\begin{array}{ll} & \text { Papelógrafo } \\
\text { s } \\
\text { - } & \text { plumones }\end{array}$ \\
\hline $15^{\prime}$ & $\begin{array}{l}\text { Compartir lo elaborado } \\
\text { y cierre de la sesión }\end{array}$ & $\begin{array}{l}\text { Realizar mini resumen } \\
\text { oral acerca del tema } \\
\text { tratado }\end{array}$ & $\begin{array}{l}\text { Se plantean algunas } \\
\text { preguntas a los asistentes } \\
\text { para asegurarse de la } \\
\text { comprensión del tema }\end{array}$ & \\
\hline
\end{tabular}




\begin{tabular}{|c|c|c|c|c|}
\hline $\begin{array}{l}\text { TIEMPO } \\
\text { Sesión } 4\end{array}$ & ACTIVIDAD & OBJETIVO & METODOLOGÍA & MATERIALES \\
\hline $5^{\prime}$ & $\begin{array}{l}\text { Toma de asistencia y } \\
\text { bienvenida al taller }\end{array}$ & $\begin{array}{l}\text { Llevar un registro de } \\
\text { asistencia }\end{array}$ & $\begin{array}{l}\text { Se tomará lista a los } \\
\text { participantes del taller en un } \\
\text { registro de asistencia y } \\
\text { posteriormente se le hará } \\
\text { entrega de una etiqueta con } \\
\text { su nombre. }\end{array}$ & $\begin{array}{ll}\text { - } & \text { Lapicero } \\
\text { - } & \text { Plumones } \\
\text { - } & \text { Etiquetas } \\
\text { - } & \text { Hoja de } \\
& \text { asistencia }\end{array}$ \\
\hline $10^{\prime}$ & $\begin{array}{l}\text { Dinámica para } \\
\text { recordatorio de sesión } \\
\text { pasada }\end{array}$ & $\begin{array}{l}\text { Recordar lo visto en la } \\
\text { sesión anterior }\end{array}$ & $\begin{array}{l}\text { Se realizan algunas preguntas } \\
\text { con respecto a lo visto en la } \\
\text { sesión anterior invitando a los } \\
\text { participantes en aportar sus } \\
\text { conocimientos }\end{array}$ & \\
\hline $10^{\prime}$ & $\begin{array}{l}\text { Exposición acerca del } \\
\text { significado de castigo }\end{array}$ & $\begin{array}{l}\text { Identificar qué es el } \\
\text { castigo }\end{array}$ & $\begin{array}{l}\text { Se expone qué es el castigo, } \\
\text { se ejemplifica. }\end{array}$ & $\begin{array}{ll}\text { - } & \text { Laptop } \\
\text { - } & \text { PPT } \\
\text { - } & \text { Proyector }\end{array}$ \\
\hline $10^{\prime}$ & $\begin{array}{l}\text { Explicar las diferencias } \\
\text { entre castigar y } \\
\text { reforzar }\end{array}$ & $\begin{array}{l}\text { Comprender la } \\
\text { diferencia entre castigo } \\
\text { y refuerzo }\end{array}$ & Se explica la diferencia & $\begin{array}{ll}\text { - } & \text { Laptop } \\
\text { - } & \text { PPT } \\
\text { - } & \text { Proyector }\end{array}$ \\
\hline $25^{\prime}$ & $\begin{array}{l}\text { Exponer tipos de } \\
\text { castigo y qué tipos de } \\
\text { conducta castigar. }\end{array}$ & $\begin{array}{l}\text { Identificar tipos de } \\
\text { castigo para cada } \\
\text { conducta }\end{array}$ & $\begin{array}{l}\text { Exposición sobre los tipos de } \\
\text { castigo positivo y negativo y } \\
\text { ejemplificar con distintas } \\
\text { conductas que suelen } \\
\text { presentar los niños. }\end{array}$ & $\begin{array}{ll}\text { - } & \text { Laptop } \\
\text { - } & \text { PPT } \\
\text { - } & \text { Proyector }\end{array}$ \\
\hline $20^{\prime}$ & $\begin{array}{l}\text { Explicar estilos de } \\
\text { castigos y manejo de } \\
\text { conductas desafiantes }\end{array}$ & $\begin{array}{l}\text { Aprender cómo castigar } \\
\text { sin violencia }\end{array}$ & $\begin{array}{l}\text { Brindar herramientas sobre } \\
\text { estilos de castigo que se } \\
\text { pueden utilizar para } \\
\text { conductas desafiantes. }\end{array}$ & $\begin{array}{ll}\text { - } & \text { Laptop } \\
\text { - } & \text { PPT } \\
\text { - } & \text { Proyector }\end{array}$ \\
\hline $20^{\prime}$ & Economía de fichas & $\begin{array}{l}\text { Aprender herramienta } \\
\text { para manejo de } \\
\text { conductas }\end{array}$ & $\begin{array}{l}\text { Explicar el cuadro de } \\
\text { economía de fichas y realizar } \\
\text { uno breve de ejemplo. }\end{array}$ & $\begin{array}{ll}\text { - } & \text { Laptop } \\
\text { - } & \text { PPT } \\
\text { - } & \text { Proyector } \\
\text { - } & \text { Hojas } \\
\text { - } & \text { Lapiceros }\end{array}$ \\
\hline $15^{\prime}$ & $\begin{array}{l}\text { Actividad de cierre de } \\
\text { la sesión }\end{array}$ & $\begin{array}{l}\text { Realizar resumen oral } \\
\text { acerca del tema tratado }\end{array}$ & $\begin{array}{l}\text { Responder dudas pendientes } \\
\text { e invitar a acercarse a los } \\
\text { consultorios en caso } \\
\text { consideren que necesitan } \\
\text { ayuda más especializada }\end{array}$ & \\
\hline
\end{tabular}




\section{APÉNDICE 3: ESQUEMA INFORME PSICOLÓGICO}

\section{INFORME PSICOLÓGICO}

\section{Datos de filiación}

- Nombre

- Sexo

- Edad

- Lugar y fecha de nacimiento

- Composición familiar

- Posición ordinal

- Centro de estudios

- Fechas de evaluación

- Informantes

- Evaluador

- Supervisor: Lic.

\section{Motivo de consulta}

Se escribe brevemente las razones por las que fue referido o de la solicitud de la evaluación.

\section{Observaciones generales}

Descripción general del físico del paciente, cómo se comportó durante el proceso de evaluación (seguía instrucciones, se concentraba, nivel de vocabulario, etc.) y la infraestructura en donde se realizó la misma.

\section{Anamnesis}

Se exploran 7 áreas: desarrollo inicial, educación escolar, educación hogareña y religión, relaciones interpersonales, historia psicosexual, hábitos y enfermedades o accidentes. Se debe redactar de forma concisa, haciendo uso de viñetas.

\section{Instrumentos utilizados}


Mencionar los instrumentos utilizados a lo largo del proceso de evaluación, haciendo uso de viñetas.

\section{Interpretación de resultados}

Se describen los resultados cualitativos y cuantitativos, considerando el área intelectual, la expresión y articulación del lenguaje, el estado de maduración social en el que se encuentra y, por último, el área emociona y su interacción familiar.

\section{Conclusiones}

Colocar en viñetas las conclusiones a las que se llega a partir de las actividades o evaluaciones realizadas.

\section{Recomendaciones}

Se redactan con viñetas las sugerencias derivadas de las conclusiones obtenidas. 